\title{
汶川-芦山地震间的空区危险性研究：现状、思考 和挑战
}

郑勇 ${ }^{1,3^{*}}$, 郭汝梦 $2,3,4$

1. 中国地质大学(武汉)地球物理与空间信息学院, 矿产资源与地质过程国家重点实验室, 武汉 430074;

2. 香港中文大学地球系统科学课程, 香港 999077 ;

3. 中国科学院精密测量科学与技术创新研究院, 武汉 430071;

4. 中国科学院大学地球与行星科学学院, 北京 100049

* 通讯作者, E-mail: zhengyong@cug.edu.cn

收稿日期: 2020-09-14; 收修改稿日期: 2020-12-28; 接受日期: 2021-01-12; 网络版发表日期: 2021-01-28 中国地震实验场项目(编号: 2019CSES0109、2018CSES0101)、国家自然科学基金项目(批准号: 41874053、42030108、41731072)和中央高校 基本科研业务项目(编号: 162301132637、CUGCJ1707)资助

\section{1 汶川-芦山地震间地震空区的研究背景}

2008 年 $M_{\mathrm{w}} 7.9$ 汶川地震和 2013 年 $M_{\mathrm{w}} 6.6$ 芦山地震, 相继发生在龙门山断裂带上，造成了惨重的人员伤亡 和经济损失. 它们的余震勾画了一个约 $50 \mathrm{~km}$ 的地震 空区(图1). 假设该空区与芦山地震有着相似的背景构 造，理论上该区域具备发生M7.0地震的可能性. 研究 该区域的发震潜力对于科学指导抗震救灾具有极为重 要的现实意义, 同时, 也可以为其他地震空区的危险性 研究提供借鉴，如鲜水河断裂带的道孚-康定段空区 (Guo等, 2020)、安宁河空区等.

\section{2 地震空区危险性的研究现状}

关于该地震空区短期内会不会发生中强地震，目 前存在着非常激烈的争论. 不同学者分别从地震学、 大地测量学、地质学等多种学科和角度, 对该区域的 地震危险性进行了全方位的研究. 总体来看, 存在着 两种截然不同的观点，一种认为该空区发生较强地震
的可能性不大(如Li等, 2013; Pei等, 2015; Li等, 2018); 另一种观点认为该区域发生中强地震的可能性很大 (如Wang等, 2015; Diao等, 2018; Liu等, 2018; Wang等, 2018; Guo等, 2020). 下面分别对这两种观点的研究进 行介绍.

( i ) 否定该空区具备发生中强地震的可能性

基于长期的GPS震间观测, Li等(2018)发现汶川地 震后, 该空区解耦, 平均耦合系数下降到 0.2 以下, 中强 地震的发震潜力比较小, 具有较强危险性的是未破裂 的龙门山断裂系统的西南端. 除此之外, 基于地壳速 度结构的特征,一些学者也认为该区域危险性不强. Li等(2013)和Pei等(2015)利用体波成像的方法研究了 龙门山地区的速度结构, 发现汶川地震和芦山地震的 发震区域都处于高速异常区，暗示该区域的地震主要 发生在强度较大的高速区域. 而汶川-芦山空区位于低 速异常区, 他们认为该区域强度较低, 不足以发生较强 地震.

(ii) 支持该空区具备发生较大地震的危险性 与前面的观点相反, 目前很多研究都表明该空区 


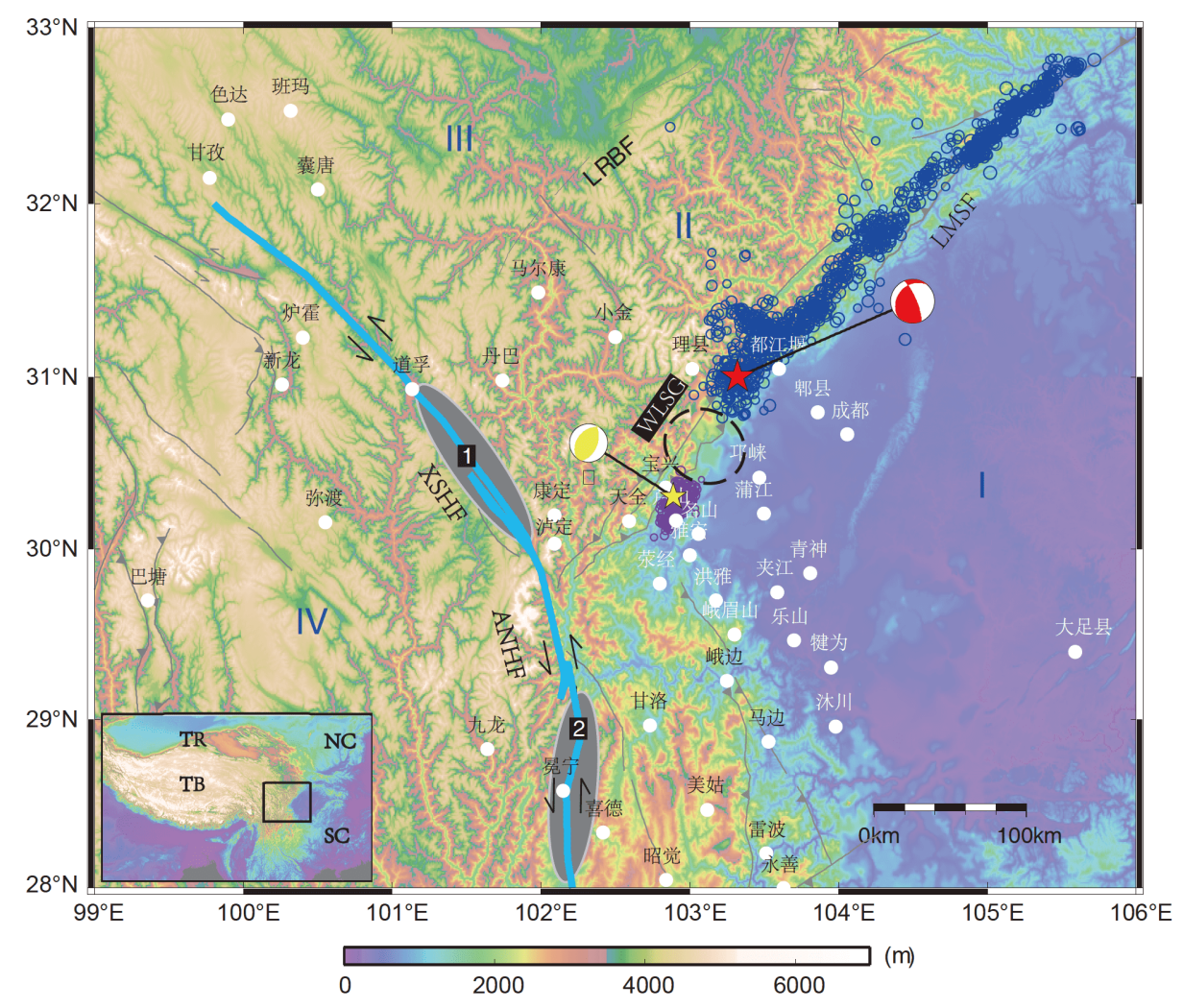

图 1 汶川地震和芦山地震之间地震空区的构造背景

红色和黄色的沙滩球分别代表汶川地震和芦山地震的震源机制. 红色和黄色的五角星分别代表汶川地震和芦山地震的震中位置. 蓝色和紫色 的圆圈分别代表汶川地震和芦山地震余震重定位的结果. 深灰色的椭圆1和2分别代表鲜水河中南段 (Guo等, 2020)和安宁河地震空区. 白色实 心圆圈代表周边的城市分布. WLSG: 汶川地震和芦山地震之间的地震空区. LMSF: 龙门山断层; MJF: 峎江断层; LRBF: 龙日坝断层; XSHF: 鲜水河断层; ANHF: 安宁河断层. I: 华南地块; II: 龙门山次级块体; III: 阿坝次级块体; IV: 川滇北部次级块体

的地震危险性非常高. Wang等(2015)通过GPS速度在 该地震空区观测到了明显的地震矩亏损，认为其具备 孕育强震的能力; Wang等(2018)发现在该地震空区内, 微震活动性相对南北两侧很弱, 表明积累的应力应变 通过微震释放出去的可能性很小.Diao等(2018)通过 研究汶川地震的震后机制, 发现在该空区内几乎没有 余滑分布，并且通过空区内的GPS观测发现其仍然在 积累应变，推测该空区有着比较大的地震危险性. 另 外，基于库伦应力的研究发现，汶川地震和芦山地震 大大增强了该空区的应力，从而使该区域发生强震的 可能性大大增加(Guo等, 2020).

\section{3 目前研究存在的局限性}

目前对地震空区的地震危险性研究存在着一定的 局限性: 首先, 大多数工作都是基于某一种手段或者研
究对象开展的, 比如速度结构、应变率积累及释放、 古地震活动等，从而导致了不唯一的结果. 如， $\mathrm{Li}$ 等 (2018)通过GPS震间形变反演的断层耦合系数, 表明 汶川-芦山地震空段危险性不高; Wang等(2015)和Diao 等(2018)则通过估计释放的应变大小来判断该区域是 否存在滑移亏损, 未释放的地震矩越大, 孕震能力越 强. 然而, 如果一个区域的闭锁程度比较低, 那么即使 应变的积累比较高, 依然可能通过无震滑动等方式释 放; 反过来, 如果应变积累足够高, 哪怕耦合系数比较 小, 仍然存在发生强震的可能性. 此外, 层析成像存在 动力学上的认识不足而导致判断的差异, 虽然目前大 多数层析成像结果表明龙门山断裂系统的地震主要发 生在高速区域, 地震空区主要位于低速区域, 其强度不 足以孕育强震(如Li等, 2013; Pei等, 2015). 然而, 古地 震研究发现该空段历史上曾经发生过6.5 7.0级地震, 因此, 低速区域可能会发生强震(Dong等, 2017). 这两 
者的矛盾可能来自于对孕震环境的认识，以及介质的 速度与强度的关系上. 虽然龙门山断裂带区域大部分 强震发生在强度较高的区域，但是汶川地震破裂区域 的南北两端速度也相对较低. 同时, 由于地壳的复杂 性，速度相对较低的断裂带区域，其强度不一定偏低， 与孔隙度、破碎程度、化学组分密切相关(Roland等, 2012), 因而也具备发生中强地震的可能. 另外, 考虑到 地震动力学因素, 如果一个断层的跨度较大, 深度较 深，那么在强度大的区域起破后，在破裂作用下发生 级联破裂，有可能导致强度较低的断层区域也发生破 裂，形成较大的地震，从而造成古地震观测与基于速 度结构判断的差异.

其次，中小地震活动性分布不能完全判断闭锁状 态, 地震平静的区域也可能是由于无震滑动(如余滑、 慢滑移、震颤等)或者微震释放了累积的应力, 从而降 低了地震潜力(Wang等, 2018).

第三, 该区域的地形差异很大, 而地形导致的正应 力分布通常与同震滑移有着密切的关系. 在汶川地震 的两个破裂剖面上，应力小的区域，其平均破裂滑移 量为 $5 \mathrm{~m}$, 而应力大的区域平均滑移量为 $3 \mathrm{~m}, 1.0 \mathrm{MPa}$ 的 正压力产生了近 $2 \mathrm{~m}$ 的破裂滑移量的差异( Tan 等, 2018). 忽略外力因素的影响，地震危险性评估也许就 会出现较大偏差. 因此, 综合考虑多种因素和物理过程 是准确评估地震空区危险性的关键.

\section{4 思考和挑战}

如前所述，单一的研究对象和方法难以得到可靠 的结果. 因此, 准确评估地震空区危险性就需要针对 目前研究存在的不足, 综合考虑孕震构造、断层运动 及闭锁状态、古地震的复发周期、背景构造应力、地 形应力、地震和无震滑动产生的应力以及动力学过程 等多个方面的因素. 因此, 我们提出了如下的研究思路 (图2): (1) 基于密集的地震、重力等数据, 确定断层及 周边区域的精细地壳结构; (2) 基于地震观测确定中小 地震的分布和震源性质, 了解地震活动性和迁移情况, 阐明断层的几何特征和发震构造; (3) 基于近断层密集 台阵探测及测井、岩石学等数据, 获取可能的断层带 的物理性质(例如静摩擦和滑动摩擦系数、材料属 性、断层破碎带性质等); (4) 基于震间的大地测量形 变数据, 确定断层的滑移状态及亏损情况, 确定断层的 闭锁程度； (5) 结合地震、大地测量、野外观测等资 料, 确定空区附近历史中强震的破裂过程和震后形变, 计算它们对断层库伦应力场的影响; (6) 综合利用大地 电磁、大地测量等数据探索下地壳以及上地幔的流变 性质，更加合理地评估黏弹性质对地震周期过程中应 力应变的影响；(7) 基于地理数据估计地形在断层面 上的正应力, 识别出阻碍破裂的应力障碍体; (8) 综合 多方面的已知信息，基于断层的物理性质和合理的应

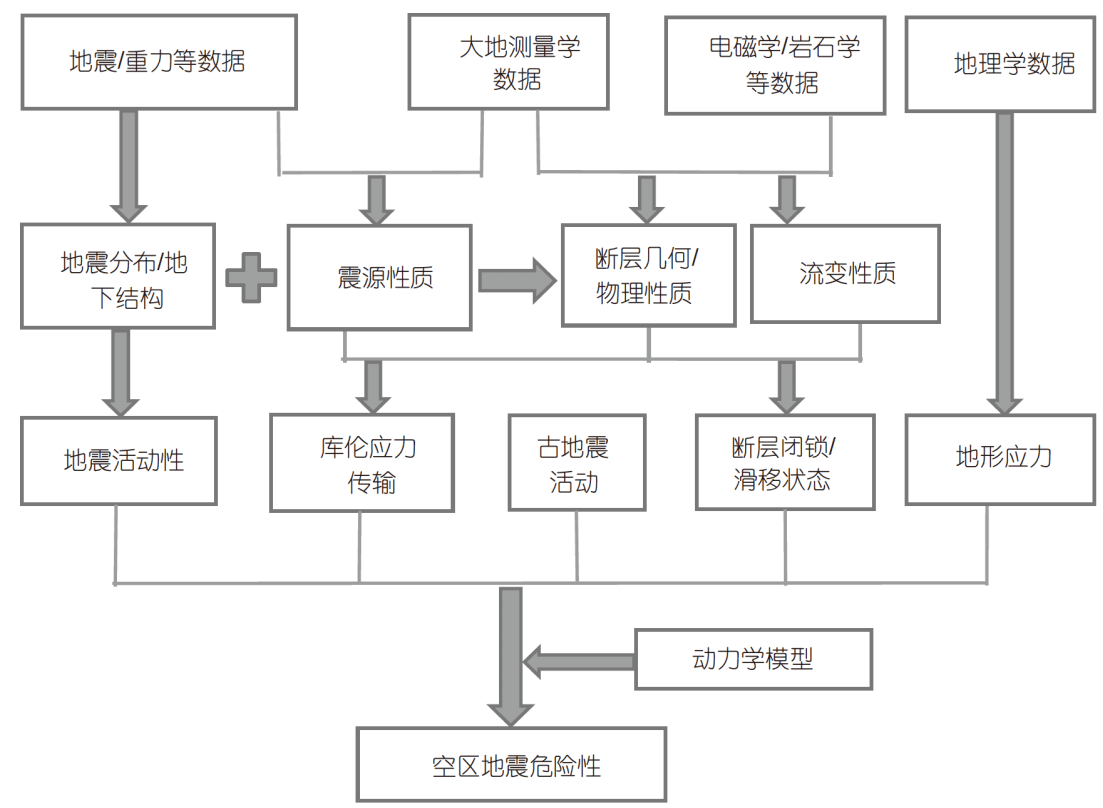

图 2 地震空段危险性研究的技术路线图 
力加载及边界约束, 建立断层系统的动力学模型, 计算 断层面及周边的应力场，分析从孕震过程到失稳破裂 的物理问题，定量评估地震空区的地震潜力，分析其 未来中强地震的发震模式.

不过, 这些研究需要面对以下的挑战:

（1）精确的断层几何及物理性质. 断层的几何结构 和精确位置是控制地震破裂过程和危险性大小的关 键. 然而断层的性质通常比较复杂, 需要结合密集台网 观测和测井、岩石学等进行综合分析.

(2) 断层闭锁状态的分析. 断层闭锁状态不仅与地 震活动性有关, 还与无震滑动密切相关; 另外, 地表形 变主要对断层浅部的滑移比较敏感, 深部的状态则需 要结合中小地震以及微震的分布和迁移来判定. 因此, 只有结合大地测量和密集的地震台阵进行综合分析, 才能给出可靠的闭锁状态.

（3）非线性介质性质对断层滑移速率和闭锁程度 的影响. 介质的黏弹性性质对断层的滑移和闭锁有较 大影响，纯弹性模型会产生较大的偏差，特别是对闭 锁时间较长, 地壳强度相对比较低的区域, 例如龙门 山断裂带、鲜水河断裂带等区域，在分析断层滑移、 闭锁状态，以及震后形变时，介质的黏弹性效应不能 忽略. 因此, 需要对研究区域的流变性质有比较深入 的认识.

(4) 较为全面的的库伦应力分析. 在分析地震空区 受周边地震应力触发时，不仅需要考虑中强地震的同 震应力影响，震后形变、强余震，以及历史地震的作 用也不可忽略，这需要对历史地震震源性质和震后机 制有较为完整的了解. 此外, 地形、三维结构、以及 气候和人类活动的影响，如页岩气的开采、流体导致 的介质孔隙饱和度变化等因素，都将是未来地震空区 危险性研究需要思考的问题和挑战. 总体而言, 随着 地震和大地测量观测技术的提高，以及对断层闭锁和 动力学机理认识的深入, 定量评估包括汶川-芦山地震 空区、道孚-康定空区等典型地震空区的地震危险性 已经成为可能.

致谢 我们对主编、编委以及两位若名审稿人提出的宝 贵意见表示衷心的感谢。

\section{参考文献}

Diao F, Wang R, Wang Y, Xiong X, Walter T R. 2018. Fault behavior and lower crustal rheology inferred from the first seven years of postseismic GPS data after the 2008 Wenchuan earthquake. Earth Planet Sci Lett, 495: 202-212

Dong S, Han Z, An Y. 2017. Paleoseismological events in the "seismic gap" between the 2008 Wenchuan and the 2013 Lushan earthquakes and implications for future seismic potential. J Asian Earth Sci, 135: $1-15$

Guo R, Zheng Y, Xu J. 2020. Stress modulation of the seismic gap between the $2008 M_{\mathrm{s}} 8.0$ Wenchuan earthquake and the $2013 M_{\mathrm{s}} 7.0$ Lushan earthquake and implications for seismic hazard. Geophys J Int, 221: 2113-2125

Li Y, Zhang G, Shan X, Liu Y, Wu Y, Liang H, Qu C, Song X. 2018. GPS-derived fault coupling of the Longmenshan Fault associated with the $2008 M_{\mathrm{w}}$ Wenchuan 7.9 Earthquake and its tectonic implications. Remote Sens, 10: 753

Li Z, Tian B, Liu S, Yang J. 2013. Asperity of the 2013 Lushan earthquake in the eastern margin of Tibetan Plateau from seismic tomography and aftershock relocation. Geophys J Int, 195: 2016-2022

Liu Z, Liang C, Hua Q, Li Y, Yang Y, He F, Fang L. 2018. The seismic potential in the seismic gap between the Wenchuan and Lushan earthquakes revealed by the joint inversion of receiver functions and ambient noise data. Tectonics, 37: 4226-4238

Pei S, Zhang H, Su J, Cui Z. 2015. Ductile gap between the Wenchuan and Lushan earthquakes revealed from the two-dimensional Pg seismic tomography. Sci Rep, 4: 6489

Roland E, Lizarralde D, McGuire J J, Collins J A. 2012. Seismic velocity constraints on the material properties that control earthquake behavior at the Quebrada-Discovery-Gofar transform faults, East Pacific Rise. J Geophys Res, 117: B11102

Tan X, Yue H, Liu Y, Xu X, Shi F, Xu C, Ren Z, Shyu J B H, Lu R, Hao H. 2018. Topographic loads modified by fluvial incision impact fault activity in the Longmenshan thrust belt, eastern margin of the Tibetan Plateau. Tectonics, 37: 3001-3017

Wang C, Liang C, Deng K, Huang Y, Zhou L. 2018. Spatiotemporal distribution of microearthquakes and implications around the seismic gap between the Wenchuan and Lushan earthquakes. Tectonics, 37: 2695-2709

Wang F, Wang M, Wang Y, Shen Z K. 2015. Earthquake potential of the Sichuan-Yunnan region, western China. J Asian Earth Sci, 107: $232-243$ 